\title{
Evaluation of Antioxidant, Lipid Peroxidation and Toxic Effects after Pomegranate Intake in Healthy Human Volunteers
}

\author{
Padippurayil Faizal', Balasubramanian Satheesan², Bhavya Vinod ${ }^{3}$, \\ Kunnethedam Thomas Augusti ${ }^{*}$
}

\begin{abstract}
${ }^{1}$ Department of Medical Biochemistry, School of Health Sciences, Kannur University Thalassery Campus, Thalassery, India ${ }^{2}$ Department of Surgical Oncology, Malabar Cancer Centre, Thalassery, India

${ }^{3}$ Department of Medical Microbiology, School of Health Sciences, Kannur University Thalassery Campus, Thalassery, India Email: faizal_tly@yahoo.com, gabas9@rediffmail.com, bhavyavinod14@yahoo.com, ^drktaugusti@yahoo.com
\end{abstract}

How to cite this paper: Faizal, P., Satheesan, B., Vinod, B. and Augusti, K.T. (2017) Evaluation of Antioxidant, Lipid Peroxidation and Toxic Effects after Pomegranate Intake in Healthy Human Volunteers. International Journal of Clinical Medicine, 8 , $12-20$.

http://dx.doi.org/10.4236/ijcm.2017.81002

Received: November 26, 2016

Accepted: January 19, 2017

Published: January 22, 2017

Copyright ( 92017 by authors and Scientific Research Publishing Inc. This work is licensed under the Creative Commons Attribution International License (CC BY 4.0). http://creativecommons.org/licenses/by/4.0/

\begin{abstract}
Studies have extensively demonstrated the activation of enzymic and nonenzymic antioxidants as well as decrease of lipid peroxidation status after pomegranate intake without any observable toxicity in animal models. Comparing to animal model studies, human trials are less done on these aspects. Cellular damage caused by reactive oxygen species appears to be a major contributor in ageing and other degenerative diseases such as cancer, cardiovascular diseases, cataracts, compromised immune system, rheumatoid arthritis and brain dysfunction. Several literatures show that pomegranate has been used as a folk medicine from ancient times itself. As per the reviews, pomegranate fruit possesses antioxidant, anti-inflammatory, antiatherogenic and antitoxic effects. The primary objective of this study was to evaluate the circulatory levels of selected antioxidant and lipid peroxidation marker in healthy human volunteers before and after pomegranate intake for a period of 0 - 90 days. As the secondary objective of the study, we also assessed the toxic effects of pomegranate supplementation by determining Hepatobiliary and Renal function tests. The final result showed that majority of the study population showed a significant increase in enzymic and non-enzymic antioxidants and a decrease in oxidative stress after pomegranate intake without any observable toxicity in liver function and renal function. The significant healthy variations were more after the intake of pomegranate for a period of 90 days than that of 45 days.
\end{abstract}

\section{Keywords}

Oxidative stress, Reactive Oxygen Species, Phytochemicals, Pomegranate 


\section{Introduction}

Epidemiologic studies have shown significant inverse relationship between the incidence of various diseases and dietary intake of fruits and vegetables [1]. Free radicals have been implicated in the pathogenesis of many diseases. There is a growing evidence that excessive production of free radicals can cause or exacerbate many human diseases [2] [3]. Pomegranate has been used in folk medicine from ancient times owing to its potential health benefits [4]. The various phytochemicals present in this fruit are potent antioxidants and anti-inflammatory agents, thereby counteracting oxidative damage and inflammation which is responsible for the pathogenesis of various diseases [5] [6] [7]. The antioxidant property of pomegranate juice was shown to be three times higher than that of red wine and green tea based on the evaluation of the free radical scavenging and iron reducing capability of the juices [5]. It possesses significantly higher levels of antioxidants in comparison with the commonly consumed fruit juices [8] [9]. The major antioxidant polyphenols present in the pomegranate fruit include the ellagitannins and anthocyanins [10]. Punicalagin is the major ellagitannin present in the fruit [11]. Clinical studies with relation to this fruit are comparably lesser with that of animal trials. As there are only limited, but promising human data, the present study aimed to further strengthen support for the unique antioxidant and antiperoxidative properties of this healthy fruit. The primary objective of this study was to evaluate the circulatory levels of selected antioxidant and lipid peroxidation marker in healthy human volunteers before and after pomegranate intake for a period of $0-90$ days. As the secondary objective of the study, we also assessed the toxic effects of pomegranate supplementation by determining hepatobiliary and renal function tests.

\section{Materials and Methods}

The work has been carried out at the Dept. of Medical Biochemistry, School of Health Sciences, Kannur University. Ethical committee clearance has been obtained for the conduct of this study. An informed consent was collected from each participant prior to the study. A clinical proforma was given to each participant to collect data such as height, weight, sex, dietary pattern and previous history of illness. A total number of 40 healthy human volunteers in each group (age group of 18 - 65 yrs) as adjudged from their clinical features were selected for the study. They were divided into three groups as Group Ia-Healthy human volunteers before pomegranate intake, Group $\mathrm{Ib}-$ Healthy Human volunteers after 45 days of pomegranate intake and Group Ic-healthy human volunteers after 90 days of Pomegranate intake. Pomegranate fresh fruit was purchased from the local market of thalassery, kannur dist, kerala from where which the people belong to the study normally purchase the fruits from. Dosage of the fruit was fixed in such a manner that prior to this study another trial was conducted in which varying doses of pomegranate were given to healthy human volunteers, the varying doses were $50 \mathrm{~g} /$ day, $100 \mathrm{~g} /$ day, $150 \mathrm{~g} /$ day and $200 \mathrm{~g} /$ day out of these 
groups a a significant percentage difference in antioxidant activity and antiperoxidative activity were shown in groups which consumed at least $100 \mathrm{~g} /$ day and more, so in this study $200 \mathrm{~g} /$ day was given, which showed maximal activity among the varying dosages used for the study in the previous trials. Participants suffering from any diseases/disorders that may interfere with the study were excluded. $5 \mathrm{~mL}$ of blood sample was taken for the analysis. Study was designed for a period of 90 days. Blood samples were collected before the intake, 45 days after the intake and 90 days after the intake of fresh pomegranate fruit at a dosage of $200 \mathrm{~g} /$ day. Serum/Plasma was separated and estimated using the standard methods. The major enzymic antioxidants analysed were Catalase (Maehly and Chance) [11], Superoxide dismutase (Marklund \& Marklund) [12], Glutathione peroxidase (Paglia \& Valentine) [13], Glutathione reductase (Goldberg and Spooner) [14] and glutathione-S-transferase (Beutler) [15]; the major non-enzymic antioxidants determined were glutathione (DTNB) [16], Vitamin C (2,6-Dichlorophenol Indophenol) [17] \& Vitamin E (Baker \& Frank) [17]. The lipid peroxidation marker estimated was Malondialdehyde (Beuge et al.) [18]. For determining the toxic effects of the fruit, if any, Hepatobiliary function was assessed by determining the levels of Total Bilirubin (Jendrassik \& Grof) [19], Total Protein (Biuret) [20], Albumin (BCG) [20], Globulin (Friedwald's formulae), AST (UV, Kinetic) [21], ALT (UV, Kinetic) [21] and ALP (pNPP-AMP) [21]; Renal function was assessed by estimating the levels of Urea (GLDH-Urease) [22], Uric acid (Uricase/peroxidase) [22] and Creatinine (Jaffes) [22] according to the methods mentioned in the parenthesis by authors names. The data was analysed by using sigmaplot 13 version. $\mathrm{P}<0.05$ that was treated has statistically significant.

\section{Results}

Inter group comparison of serum SOD levels (Table $1 \&$ Table 2 ) shows a statistically significant increase between Group Ia and Ib $(\mathrm{P}<0.003)$ with a highly significant increase between Group Ia and Ic \& Group Ib and Ic $(\mathrm{P}<0.001)$. The percentage increase was more in between Initial and final levels followed by Mid and final level \& Initial and Mid level in the order $12.8>8>4.4$.

Inter group comparison of serum CAT levels (Table $1 \&$ Table 2 ) shows a statistically significant increase between Group Ia and Ib $(\mathrm{P}<0.049)$ with a highly significant increase between Group Ia and Ic \& Group Ib and Ic $(\mathrm{P}<0.001)$. The percentage increase was more in between Initial and final levels followed by Mid and final level \& Initial and Mid level in the order $62.4>37.9>17.7$.

Inter group comparison of serum Glutathione peroxidase levels (Table $1 \&$ Table 3) shows a statistically significant increase between Ib and Ic $(\mathrm{P}<0.005)$ with a highly significant increase between Group Ia and Ic $(\mathrm{P}<0.001)$. The percentage increase was more in between Initial and final levels followed by Mid and final level \& Initial and Mid level in the order $23.9>14.3>8.3$. Even though there is no statistically significant change between Group Ia and Ib there is a dif- 
ference in the mean percentage increase of value.

Inter group comparison of serum Glutathione reductase levels (Table 1 \& Table 3$)$ shows a statistically significant increase between $\mathrm{Ib}$ and $\mathrm{Ic}(\mathrm{P}=0.005)$ with a highly significant increase between Group Ia and Ic $(\mathrm{P}<0.001)$. The percentage increase was more in between Initial and final levels followed by Mid and final level \& Initial and Mid level in the order $24.4>15.2>8.0$. Eventhough there is no statistically significant change between Group Ia and Ib there is a difference in the mean percentage increase of value.

Inter group comparison of serum Glutathione $S$ transferase levels (Table 1 \& Table 3 ) shows a statistically significant increase between $\mathrm{Ib}$ and $\mathrm{Ic}(\mathrm{P}=0.049)$ with more significant increase between Group Ia and Ic $(P=0.004)$. The percentage increase was more in between Initial and final levels followed by Mid and final level \& Initial and Mid level in the order $11.3>7.4>3.6$. Eventhough there is no statistically significant change between Group Ia and Ib there is a difference in the mean percentage increase of value.

Inter group comparison of serum Glutathione levels (Table 4 \& Table 5) shows a statistically significant increase between $\mathrm{Ib}$ and $\mathrm{Ic}(\mathrm{P}=0.01)$ with a highly significant increase between Group Ia and Ic $(P<0.001)$. The percentage increase was more in between Initial and final levels followed by Mid and final level \& Initial and Mid level in the order $21.0>12.5>7.5$. Even though there is no statistically significant change between Group Ia and Ib there is a difference in the mean percentage increase of value.

Inter group comparison of serum Vitamin C levels (Table 4 \& Table 5) shows a statistically significant increase between $\mathrm{Ib}$ and Ic $(\mathrm{P}=0.001)$ with a highly significant increase between Group Ia and Ic $(\mathrm{P}<0.001)$. The percentage increase was more in between Initial and final levels followed by Mid and final level \& Initial and Mid level in the order $29.0>18.7>8.7$. Even though there is no statistically significant change between Group Ia and Ib there is a difference in the mean percentage increase of value

Inter group comparison of serum Vitamin E levels (Table 4 \& Table 5) shows a statistically significant increase between Group $\mathrm{Ia}$ and $\mathrm{Ib}(\mathrm{P}=0.001)$, with a highly significant increase between Ia and Ic \& Ib and Ic $(P<0.001)$. The percentage increase was more in between Initial and final levels followed by Mid and final level \& Initial and Mid level in the order $25.7>17.8>8.5$.

Inter group comparison of serum MDA levels (Table $4 \&$ Table 6) shows a statistically highly significant decrease in all groups studied $(\mathrm{P}<0.001)$. The mean percentage decrease was more in between Initial and final levels followed by Mid and final level \& Initial and Mid level in the order $-17.4>-12.4>-5.7$.

Inter group comparison of Liver function parameters viz; serum Total Bilirubin, Total Protein, Albumin and Globulin, AST, ALT, ALP (Table 7, Table 8, Table 9 \& Table 10) and renal function parameters viz; Blood urea, serum uric acid and creatinine (Table $11 \&$ Table 12 ) were statistically insignificant in all groups studied, showing that supplementation of pomegranate does not have any toxic effects for the organs liver and kidney. 
Table 1. Mean \pm SD levels of enzymic antioxidants in Group I, before and after supplementation of pomegranate fruits.

\begin{tabular}{ccccccc}
\hline & \multicolumn{5}{c}{ Parameter } \\
\cline { 3 - 7 } Mean \pm SD & SOD (U/mL) & CAT (IU/L) & GPX (U/L) & GR (IU/L) & GST (IU/L) \\
\cline { 3 - 7 } & R.R $=$ & R.R $=$ & R.R $=$ & R.R $=$ & R.R $=$ \\
& $2.80-3.95$ & $10-50$ & $95-206$ & 30 to 80 & $4.30-6.40$ \\
\hline \multirow{2}{*}{ Group I } & 45 day (b) & $3.27 \pm 0.16$ & $21.9 \pm 6.3$ & $161.34 \pm 26.69$ & $53.58 \pm 10.54$ & $5.15 \pm 0.57$ \\
& 90 day (c) & $3.53 \pm 0.20$ & $30.2 \pm 6.0$ & $184.49 \pm 28.48$ & $61.75 \pm 8.68$ & $5.53 \pm 0.69$ \\
\hline
\end{tabular}

Table 2. Inter Group comparison, $t$ value, $p$ value and mean difference $\%$ of SOD and CAT.

\begin{tabular}{ccccccc}
\hline \multirow{2}{*}{ Groups } & \multicolumn{3}{c}{ SOD } & \multicolumn{3}{c}{ CAT } \\
\cline { 2 - 7 } & t value & p value & MD \% & t value & p value & MD \% \\
\hline IaVsIb & 3.031 & 0.003 & 4.4 & 2.004 & 0.049 & 17.7 \\
IaVsIc & 8.660 & $<0.001$ & 12.8 & 7.044 & $<0.001$ & 62.4 \\
IbVsIc & 5.629 & $<0.001$ & 8 & 5.040 & $<0.001$ & 37.9 \\
\hline
\end{tabular}

Table 3. Inter Group comparison, $t$ value, $p$ value and mean difference $\%$ of GPX, GR and GST.

\begin{tabular}{lccccccccc}
\hline \multirow{2}{*}{ Groups } & \multicolumn{3}{c}{ GPX } & \multicolumn{3}{c}{ GR } & \multicolumn{3}{c}{ GST } \\
\cline { 2 - 10 } & t value & p value & MD \% & t value & p value & MD \% & t value & p value & MD \% \\
\hline IaVsIb & 1.680 & $0.097^{*}$ & 8.3 & 1.509 & $0.136^{*}$ & 8 & 1.085 & $0.281^{*}$ & 3.6 \\
IaVsIc & 4.814 & $<0.001$ & 23.9 & 4.621 & $<0.001$ & 24.4 & 3.377 & 0.004 & 11.3 \\
IbVsIc & 3.134 & 0.005 & 14.3 & 3.113 & 0.005 & 15.2 & 2.291 & 0.049 & 7.4 \\
\hline
\end{tabular}

Table 4. Mean \pm SD levels of non-enzymic antioxidants \& MDA in Group I before and after supplementation of pomegranate fruits.

\begin{tabular}{cccccc}
\hline & \multicolumn{4}{c}{ Parameter } \\
\cline { 3 - 6 } Mean \pm SD & GSH $(\mathrm{mg} / \mathrm{dL})$ & Vit. C $(\mathrm{mg} / \mathrm{dL})$ & Vit. E $(\mathrm{mg} / \mathrm{L})$ & MDA $(\mathrm{nmol} / \mathrm{mL})$ \\
\cline { 3 - 6 } & R.R $=15-55$ & R.R $=0.6-1.4$ & R.R $=6-19$ & R.R $=8-20$ \\
\hline \multirow{2}{*}{ Group I } & 45 day (b) & $25.28 \pm 3.82$ & $0.75 \pm 0.16$ & $12.15 \pm 1.04$ & $7.97 \pm 0.26$ \\
& 90 day (c) & $28.45 \pm 4.20$ & $0.89 \pm 0.13$ & $14.32 \pm 1.10$ & $6.98 \pm 0.20$ \\
\hline
\end{tabular}

Table 5. Inter Group comparison, $t$ value, $p$ value and mean difference $\%$ of Glutathione, Vit.C and Vit.E.

\begin{tabular}{cccccccccc}
\hline \multirow{2}{*}{ Groups } & \multicolumn{3}{c}{ Glutathione } & \multicolumn{3}{c}{ Vitamin C } & \multicolumn{3}{c}{ Vitamin E } \\
\cline { 2 - 9 } & $\mathrm{t}$ value & $\mathrm{p}$ value & $\mathrm{MD} \%$ & $\mathrm{t}$ value & $\mathrm{p}$ value & $\mathrm{MD} \%$ & $\mathrm{t}$ value & $\mathrm{p}$ value & $\mathrm{MD} \%$ \\
\hline IaVsIb & 1.625 & $0.108^{*}$ & 7.5 & 1.540 & $0.128^{*}$ & 8.7 & 3.303 & 0.001 & 8.5 \\
IaVsIc & 4.536 & $<0.001$ & 21 & 5.134 & $<0.001$ & 29 & 10.849 & $<0.001$ & 25.7 \\
IbVsIc & 2.911 & 0.010 & 12.5 & 3.594 & 0.001 & 18.7 & 7.545 & $<0.001$ & 17.8 \\
\hline
\end{tabular}


Table 6. Inter Group comparison, $\mathrm{p}$ value, $\mathrm{t}$ value and mean difference $\%$ of MDA.

\begin{tabular}{cccc}
\hline \multirow{2}{*}{ Groups } & \multicolumn{3}{c}{ MDA } \\
\cline { 2 - 4 } & $\mathrm{t}$ value & $\mathrm{p}$ value & $\mathrm{MD} \%$ \\
\hline IaVsIb & 6.128 & $<0.001$ & -5.7 \\
IaVsIc & 18.766 & $<0.001$ & -17.4 \\
IbVsIc & 12.638 & $<0.001$ & -12.4 \\
\hline
\end{tabular}

Table 7. Mean \pm SD levels of Liver function parameters in Group I, before and after supplementation of Pomegranate fruits; (b) Mean \pm SD levels of Liver function parameters in Group I, before and after supplementation of pomegranate fruits.

(a)

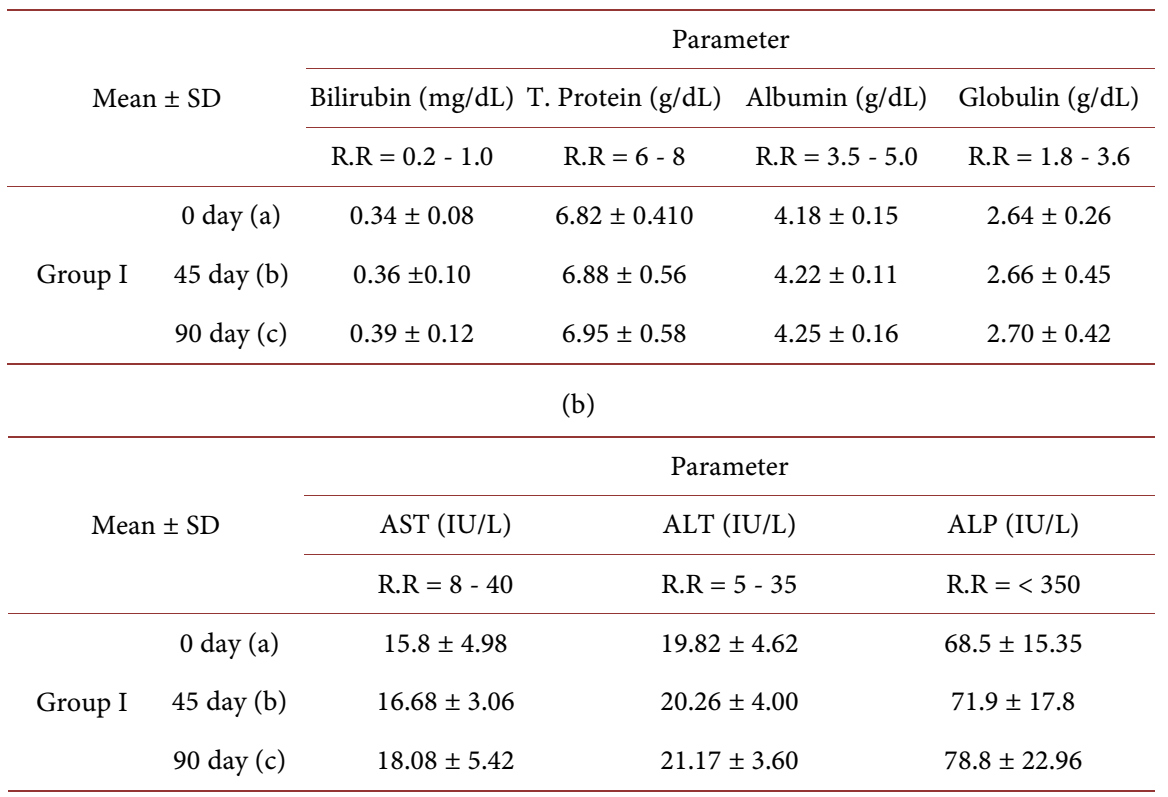

Table 8. Inter group comparison, $\mathrm{t}$ value, $\mathrm{p}$ value and mean difference $\%$ of Bilirubin and Total Protein.

\begin{tabular}{ccccccc}
\hline \multirow{2}{*}{ Groups } & \multicolumn{3}{c}{ Bilirubin } & & \multicolumn{3}{c}{ T. Protein } \\
\cline { 2 - 7 } & t value & p value & MD \% & t value & p value & MD \% \\
\hline IaVsIb & 0.698 & $0.488^{*}$ & 5.8 & 0.406 & $0.686^{*}$ & 0.9 \\
IaVsIc & 1.745 & $0.235^{*}$ & 14.7 & 0.88 & $0.764^{*}$ & 1.9 \\
IbVsIc & 1.047 & $0.508^{*}$ & 8.3 & 0.474 & $0.868^{*}$ & 1 \\
\hline
\end{tabular}

Table 9. Inter group comparison, $\mathrm{t}$ value, $\mathrm{p}$ value and mean difference $\%$ of Albumin and Globulin.

\begin{tabular}{ccccccc}
\hline \multirow{2}{*}{ Groups } & \multicolumn{3}{c}{ Albumin } & \multicolumn{3}{c}{ Globulin } \\
\cline { 2 - 6 } & t value & p value & MD \% & t value & p value & MD \% \\
\hline IaVsIb & 0.998 & $0.540^{*}$ & 0.9 & 0.183 & $0.855^{*}$ & 0.8 \\
IaVsIc & 1.747 & $0.234^{*}$ & 1.7 & 0.550 & $0.928^{*}$ & 2.3 \\
IbVsIc & 0.749 & $0.456^{*}$ & 0.7 & 0.367 & $0.919^{*}$ & 1.5 \\
\hline
\end{tabular}


Table 10. Inter group comparison, $\mathrm{p}$ value, $\mathrm{t}$ value and mean difference $\%$ of AST, ALT and ALP.

\begin{tabular}{lccccccccc}
\hline \multirow{2}{*}{ Groups } & \multicolumn{3}{c}{ AST } & \multicolumn{3}{c}{ ALT } & \multicolumn{3}{c}{ ALP } \\
\cline { 2 - 10 } & t value & p value & MD $\%$ & t value & p value & MD \% & t value & p value & MD \% \\
\hline IaVsIb & 0.676 & $0.501^{*}$ & 5.6 & 0.380 & $0.705^{*}$ & 2.2 & 0.634 & $0.528^{*}$ & 4.9 \\
IaVsIc & 1.752 & $0.232^{*}$ & 14.1 & 1.166 & $0.574^{*}$ & 6.8 & 1.920 & $0.166^{*}$ & 15 \\
IbVsIc & 1.076 & $0.490^{*}$ & 8.4 & 0.786 & $0.68^{*}$ & 4.5 & 1.286 & $0.364^{*}$ & 9.6 \\
\hline
\end{tabular}

Table 11. Mean \pm SD levels of Renal function parameters in Group I, before and after supplementation of pomegranate fruits.

\begin{tabular}{lcccc}
\hline & & \multicolumn{3}{c}{ Parameter } \\
\cline { 3 - 5 } Mean \pm SD & Bl. Urea $(\mathrm{mg} / \mathrm{dL})$ & Uric acid $(\mathrm{mg} / \mathrm{dL})$ & Creatinine $(\mathrm{mg} / \mathrm{dL})$ \\
\cline { 3 - 5 } & & R.R $=15-40$ & R.R $=3.5-7.0$ & R.R $=0.6-1.4$ \\
\hline \multirow{2}{*}{ Group I } & 45 day (a) & $24.50 \pm 4.46$ & $4.18 \pm 0.95$ & $0.91 \pm 0.17$ \\
& 90 day (c) & $23.33 \pm 3.32$ & $4.46 \pm 0.820$ & $0.88 \pm 0.14$ \\
& $25.24 \pm 3.47$ & $4.62 \pm 0.68$ & $0.95 \pm 0.13$ \\
\hline
\end{tabular}

Table 12. Inter Group comparison, $t$ value, $\mathrm{p}$ value and mean difference $\%$ of Bl.Urea, Uric acid and Creatinine.

\begin{tabular}{lccccccccc}
\hline & \multicolumn{3}{c}{ Bl.Urea } & \multicolumn{3}{c}{ Uric acid } & \multicolumn{3}{c}{ Creatinine } \\
\cline { 2 - 10 } Groups & t value & p value & MD \% & t value & p value & MD \% & t value & p value & MD \% \\
\hline IaVsIb & 1.093 & $0.479^{*}$ & -4.8 & 1.201 & $0.413^{*}$ & 6.7 & 0.718 & $0.475^{*}$ & -3.3 \\
IaVsIc & 0.691 & $0.492^{*}$ & 3 & 1.888 & $0.178^{*}$ & 10.5 & 0.958 & $0.566^{*}$ & 4.4 \\
IbVsIc & 1.785 & $0.218^{*}$ & 5.2 & 0.686 & $0.495^{*}$ & 3.6 & 1.676 & $0.266^{*}$ & 7.9 \\
\hline
\end{tabular}

\section{Discussion}

Dose effects of supplementary feeding of Pomegranate in healthy human volunteers show that all enzymic and non-enzymic antioxidant levels were raised in the blood significantly for both 45 days and 90 days of feeding without any observable toxicity. When we consider the effect of feeding highly significant dose effects were observed in the group who were served pomegranate for 90 days. Pomegranate fruits used in this experiment are fully enriched with several antioxidants such as phytosterols, polyphenols, flavanoids, carotenoids and Vitamins especially E \& C [9] [10]. In addition to the above, several minerals such as iron that promote synthesis of haemoglobin and selenium,that promotes glutathione peroxidase action are also there in this fruit. The finding that 90 days of feeding with pomegranate was more effective than 45 days may be due to the fact that the human body cells are more exposed to the antioxidants enriched components of the fruit. Pomegranate contains antioxidants such as anthocyanins, ellagic acid, gallic acid, punic acid, catechin, EGCG, quercetin, rutin, apigenin and other flavanoids ellagitannins and triterpenoids such as ursolic, merlinic and ariatic acids, out of which EGCG is the strongest antioxidant with 
eight hydroxyl groups and this may be the reason that pomegranate activates the enzymic and nonenzymic antioxidants of the body and reduces lipid peroxidative effects.

\section{Conclusion}

The study has shown that consumption of $200 \mathrm{mg}$ /day of Pomegranate exerts beneficial effects in human body by increasing the antioxidant defense mechanism and reducing lipid peroxidation without any observable toxicity. Pomegranate contains various nutraceuticals which are all endowed with increasing antioxidant activity and decreasing the oxidative stress. Most of the active principles contained in the fruit must have protected the cells from oxidative stress and the insignificant change in liver function and kidney function parameters indicates that there is no toxic effect for this wonder fruit and as a result it can be consumed in day-to-day life for increasing the antioxidant potential of the cells and thereby the total health status of the body can be improved to a great extent. Further clinical studies in this regard with large sample size have to be continued in order to further strengthen the role of this fruit as an immune booster.

\section{References}

[1] Temple, N.J. (2000) Antioxidants and Disease: More Questions Than Answers. Nutrition Research, 20, 449-459. https://doi.org/10.1016/s0271-5317(00)00138-x

[2] Halliwell, B., Gutteridge, J. and Cross, C.E. (1992) Free Radicals, Antioxidants, and Human Disease: Where Are We Now? The Journal of Laboratory and Clinical Medicine, 119, 598-620.

[3] McCall, M.R. and Frei, B. (1999) Can Antioxidant Vitamins Materially Reduce Oxidative Damage in Humans? Free Radical Biology and Medicine, 26, 1034-1053. https://doi.org/10.1016/S0891-5849(98)00302-5

[4] Langlery, P. (2000) Why a Pomegranate? BJ, 321, 1153.

[5] Gil, M.I., Tomás-Barberán, F.A., Hess-Pierce, B., Holcroft, D.M. and Kader, A.A. (2000) Antioxidant Activity of Pomegranate Juice and Its Relationship with Phenolic Composition and Processing. Journal of Agricultural and Food Chemistry, 48, 4581-4589. https://doi.org/10.1021/jf000404a

[6] Nasr, C.B., Ayed, N. and Metche, M. (1996) Quantitative Determination of the Polyphenolic Content of Pomegranate Peel. Zeitschrift für Lebensmittel-Untersuchung und Forschung, 203, 374-378. https://doi.org/10.1007/BF01231077

[7] Tzulker, R., Glazer, I., Bar-Ilan, I., Holland, D., Aviram, M. and Amir, R. (2007) Antioxidant Activity, Polyphenol Content, and Related Compounds in Different Fruit Juices and Homogenates Prepared from 29 Different Pomegranate Accessions. Journal of Agricultural and Food Chemistry, 55, 9559-9570. https://doi.org/10.1021/jf071413n

[8] Rosenblat, M. and Aviram, M. (2006) Antioxidative Properties of Pomegranate: In Vitro Studies. Pomegranates: Ancient Roots to Modern Medicine, 43, 31-43.

[9] Seeram, N.P., Lee, R. and Heber, D. (2004) Bioavailability of Ellagic Acid in Human Plasma after Consumption of Ellagitannins from Pomegranate (Punica granatum L.) Juice. Clinica Chimica Acta, 348, 63-68. https://doi.org/10.1016/j.cccn.2004.04.029

[10] Azadzoi, K.M., Schulman, R.N., Aviram, M. and Siroky, M.B. (2005) Oxidative Stress 
in Arteriogenic Erectile Dysfunction: Prophylactic Role of Antioxidants. The Journal of Urology, 174, 386-393. https://doi.org/10.1097/01.ju.0000161209.39959.67

[11] Chance, B.A. and Maehlly, C. (1995) Assay of Catalase and Peroxidase. Methods in Enzymology, 2, 764-765. https://doi.org/10.1016/S0076-6879(55)02300-8

[12] Marklund, S. and Marklund, G. (1974) Involvement of the Superoxide Anion Radical in the Autoxidation of Pyrogallol and a Convenient Assay for Superoxide Dismutase. European Journal of Biochemistry, 47, 469-474. https://doi.org/10.1111/j.1432-1033.1974.tb03714.x

[13] Paglia, D.E. and Valentine, W.N. (1967) Studies on the Quantitative and Qualitative Characterization of Erythrocytes Glutathione Peroxidase. Journal of Laboratory and Clinical Medicine, 70, 158-169.

[14] Goldberg, D.M. and Spooner, R.J. (1983) Assay of Glutathione Reductase. In: Bergmeyen, H.V., Ed., Methods of Enzymatic Analysis, 3rd Edition, Vol. 3, Verlog Chemie, Deerfiled Beach, 258-265.

[15] Harvey, J.W. and Beutler, E. (1982) Binding of Heme by Glutathiones-S Transferases-A Possible Role of Erythrocyte Enzyme. Blood, 60, 1227-1230.

[16] Beutler, E. (1975) Reduced Glutathione (GSH). In: Bergmeyen, H.V., Ed., Red Blood Cell Metabolism: A Manual of Biochemical Methods, 2nd Edition, Grune and Stratton, New York, 112-114.

[17] Roe, J.H. (1961) Ascorbic Acid in Blood and Urine. In: Seligson, D., Ed., Standard Methods in Clinical Chemistry, Vol. 3, Academic Press, New York, 35-45.

[18] Beuge, J.A. and Aust, S.D. (1978) Microsomal Lipid Peroxidation. Methods in Enzymology, 105, 302-310. https://doi.org/10.1016/S0076-6879(78)52032-6

[19] Pearlman, F.C. and Lee, R.T. (1974) Detection and Measurement of Total Bilirubin in Serum, with Use of Surfactants as Solubilizing Agents. Clinical Chemistry, 20, 447-453.

[20] Koller, A. (1984) Proteins. In: Kaplan, L.A. and Pesce, A.J., Eds., Clinical Chemistry. Theory, Analysis and Correlation, Mosby, Toronto, 1268-1327.

[21] Moss, D.W. and Henderson, A.K. (1994) Clinical Enzymology. In: Burtis, C.A and Ashwood, E.R., Eds., Tietz Textbook of Clinical Chemistry, 3rd Edition, WB Saunders, Philadelphia, 617-621.

[22] Murray, R.L. (1984) Non Protein Nitrogen Compounds. In: Kaplan, L.A. and Pesce, A.J., Eds., Clinical Chemistry: Theory, Analysis and Correlation, Mosby, Toronto, 1230-1268. 
Submit or recommend next manuscript to SCIRP and we will provide best service for you:

Accepting pre-submission inquiries through Email, Facebook, LinkedIn, Twitter, etc. A wide selection of journals (inclusive of 9 subjects, more than 200 journals)

Providing 24-hour high-quality service

User-friendly online submission system

Fair and swift peer-review system

Efficient typesetting and proofreading procedure

Display of the result of downloads and visits, as well as the number of cited articles Maximum dissemination of your research work

Submit your manuscript at: http://papersubmission.scirp.org/

Or contact ijcm@scirp.org 